\title{
Projection Measurement of the Maximally Entangled N-Photon State for a Demonstration of N-Photon de Broglie Wavelength
}

\author{
F. W. $\operatorname{Sun}^{1}$, Z. Y. $\mathrm{Ou}^{1,2 *}$, and G. C. Guo ${ }^{1}$ \\ ${ }^{1}$ Key Laboratory of Quantum Information, \\ University of Science and Technology of China, \\ CAS, Hefei, 230026, the People's Republic of China \\ ${ }^{2}$ Department of Physics, \\ Indiana University-Purdue University Indianapolis \\ 402 N. Blackford Street, Indianapolis, IN 46202
}

(Dated: July 24, 2018)

\begin{abstract}
We construct a projection measurement process for the maximally entangled N-photon state (the NOON-state) with only linear optical elements and photodetectors. This measurement process will give null result for any $\mathrm{N}$-photon state that is orthogonal to the NOON state. We examine the projection process in more detail for $N=4$ by applying it to a four-photon state from type-II parametric down-conversion. This demonstrates an orthogonal projection measurement with a null result. This null result corresponds to a dip in a generalized Hong-Ou-Mandel interferometer for four photons. We find that the depth of the dip in this arrangement can be used to distinguish a genuine entangled four-photon state from two separate pairs of photons. We next apply the NOON state projection measurement to a four-photon superposition state from two perpendicularly oriented type-I parametric down-conversion processes. A successful NOON state projection is demonstrated with the appearance of the four-photon de Broglie wavelength in the interference fringe pattern.
\end{abstract}

PACS numbers: 42.50.Dv, 42.25.Hz, 03.65.Ta

\section{INTRODUCTION}

Recently, attentions [1, 2, 3, 4, 5, 6, 7, 8, 9, 10] have been focussed on the generation of the so-called NOON state in the form of

$$
|N O O N\rangle=\frac{1}{\sqrt{2}}(|N, 0\rangle+|0, N\rangle) .
$$

It has been shown [1, 2] that the NOON state has the advantage in the sensitivity of optical interferometry over a coherent state and can achieve the Heisenberg limit 11 of $1 / N$ in the accuracy of phase measurement. This is a factor of $\sqrt{N}$ improvement over the traditional standard quantum limit of $1 / \sqrt{N}$ with a coherent state. Experimental demonstration for $N=2$ was first performed by Ou et al [12] and by Rarity et al 13], and more recently by Fonseca et al 14] and by Edamatsu et al 15. The extension to $N=3$ and 4 was reported by Mitchell et al [8] and Walther et al [7]. Boto et al [16] have shown that quantum lithography with the NOON state can increase the resolution by a factor of $N$ compared to a coherent state. This is so because the NOON state shows a de Broglie wavelength of $\lambda / N$ for the $N$-photon interference. D'Angelo et al [17] demonstrated the feasibility of the scheme for $N=2$.

The general trend in preparing the NOON state is by the method of quantum interference [6, 9, 10, 18] for the cancellation of the unwanted states of $|N-1,1\rangle, \mid N-$ $2,2\rangle, \ldots$, etc. A simplest example is the Hong-Ou-

\footnotetext{
*E-mail: zou@iupui.edu
}

Mandel interferometer 18] where two photons enter a 50:50 lossless beam splitter from two input sides separately $\left(\left|\Phi_{2}\right\rangle_{\text {in }}=|1,1\rangle\right)$. The output state is a NOON state of $N=2$ :

$$
\left|\Phi_{2}\right\rangle_{\text {out }}=\frac{1}{\sqrt{2}}(|2,0\rangle+|0,2\rangle) .
$$

The disappearance of the $|1,1\rangle$ in the output state in Eq.(2) is a result of quantum two-photon interference. Unfortunately, extension to four-photon state of $\left|\Phi_{4}\right\rangle_{\text {in }}=$ $|2,2\rangle$ will not produce a four-photon NOON state but a state of 19

$$
\left|\Phi_{4}\right\rangle_{\text {out }}=\sqrt{\frac{3}{8}}(|4,0\rangle+|0,4\rangle)+\frac{1}{2}|2,2\rangle .
$$

It is impossible to take out the unwanted $|2,2\rangle$ state with just linear optical elements by quantum interference. It is important that our starting state is in the form of $|2,2\rangle$ because it is what we can have from a parametric downconversion process.

In this paper, we approach this problem from another aspect, that is, the measurement process. If our measurement only responds to the first term, i.e., the NOON state, but not to the second term, i.e., $|2,2\rangle$ in Eq.(3), we will not have the contribution from the $|2,2\rangle$ term and we effectively obtain the four-photon NOON state.

Recently, an issue has been raised about how to distinguish an entangled multi-photon state from a quantum state with photons well separated and distinguishable [20, 21]. The difference lies in the multi-photon interference: an entangled multi-photon state will give rise to the strongest multi-photon interference effect whereas a 
distinguishable multi-photon state produce less or sometimes zero interference effect. It turns out that the new projection measurement scheme that we are going to introduce is based on a multi-photon interference effect to cancel the contributions from the orthogonal states. Thus this scheme will be able to quantitatively characterize the degree of entanglement for a multi-photon state.

In the following, we will first introduce the general NOON state projection measurement. We then apply it to the $|2,2\rangle$ state which is orthogonal to the four-photon NOON state. We will show that we obtain a null result confirming the orthogonality. Next we apply the projection measurement to the state in Eq.(3) and project out the NOON state for a demonstration of four-photon de Broglie wavelength. We conclude with a discussion.

\section{NOON STATE PROJECTION MEASUREMENT}

The general idea in constructing a NOON state projection measurement is very similar to Hofmann's method [6] of creating the NOON state by super-bunching of $\mathrm{N}$ independent photons. It follows from the algebraic identity:

$$
x^{N}-y^{N}=\prod_{n=0}^{N-1}\left(x-y e^{i 2 n \pi / N}\right) .
$$

If we substitute $x, y$ by $\hat{a}_{H}, \hat{a}_{V}$ with $H, V$ representing the horizontal and vertical polarizations, we have the same equation but for the operators

$$
\hat{a}_{H}^{N}-\hat{a}_{V}^{N}=\prod_{n=0}^{N-1}\left(\hat{a}_{H}-\hat{a}_{V} e^{i 2 n \pi / N}\right) .
$$

This is possible because $\hat{a}_{H}$ and $\hat{a}_{V}$ commute. Next, we make a joint measurement of the operators $\hat{b}_{n}^{\dagger} \hat{b}_{n}$ with

$$
\hat{b}_{n} \equiv\left(\hat{a}_{H}-\hat{a}_{V} e^{i 2 n \pi / N}\right) / \sqrt{2} \quad(n=0, \ldots, N-1),
$$

the joint probability $P_{N}$ is proportional to

$$
\begin{aligned}
P_{N} & \propto\left\langle\Phi_{N}\left|\hat{b}_{N-1}^{\dagger} \ldots \hat{b}_{0}^{\dagger} \hat{b}_{0} \ldots \hat{b}_{N-1}\right| \Phi_{N}\right\rangle \\
& \left.\propto\left\langle\Phi_{N}\right| \hat{a}_{H}^{\dagger N}-\hat{a}_{V}^{\dagger N}\right)\left(\hat{a}_{H}^{N}-\hat{a}_{V}^{N}\right)\left|\Phi_{N}\right\rangle
\end{aligned}
$$

where

$$
\left|\Phi_{N}\right\rangle=\sum_{n=0}^{N} c_{n}|N-n, n\rangle
$$

is an arbitrary N-photon state for two modes of $\hat{a}_{H}$ and $\hat{a}_{V}$. It is easy to see that only terms with $n=0, N$, i.e., only the NOON state part in the general state in Eq. (8) contribute to $P_{N}$ in Eq.(7). Thus we achieve a NOON state projection measurement and

$$
P_{N} \propto\left|c_{0}-c_{N}\right|^{2}
$$

If we introduce a phase shift between $\hat{a}_{H}$ and $\hat{a}_{V}$, i.e., $\varphi=\varphi_{H}-\varphi_{V}, c_{0}^{*} c_{N}=\left|c_{0} c_{N}\right| e^{i N \varphi}$ and Eq. (9) becomes

$$
P_{N} \propto\left|c_{0}\right|^{2}+\left|c_{N}\right|^{2}-2\left|c_{0} c_{N}\right| \cos N \varphi
$$

Here $\varphi$ is single photon phase difference. For the special case of $\left|c_{0}\right|=\left|c_{N}\right|$, we have

$$
P_{N} \propto 1-\cos N \varphi
$$

The dependence on $N \varphi$ is a signature of $\mathrm{N}$-photon de Broglie wave.

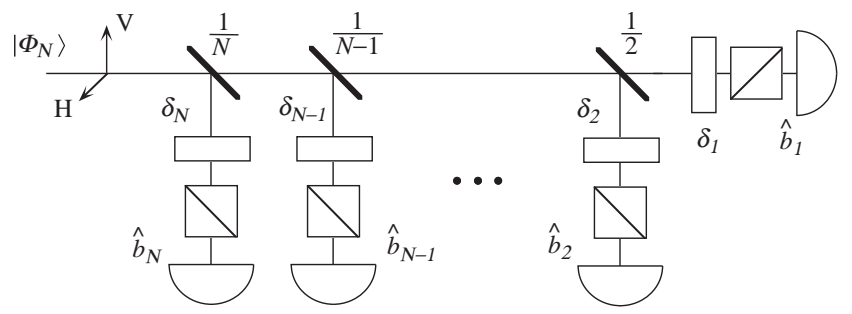

FIG. 1: Layout for the NOON state projection measurement. The number above each beam splitter is the reflectivity. $\delta_{k}=$ $2(k-1) \pi / N$ is the phase delay between $H$ and $V$ polarizations. The polarizers are 45 degree oriented.

It is straightforward to achieve the projection measurement discussed above. Consider the scheme depicted in Fig.1. It is easy to show that the operator in front of each detector has the form given in Eq.(6). Thus the n-th detector measures $\hat{b}_{n}^{\dagger} \hat{b}_{n}$. The N-fold coincidence measurement of all $\mathrm{N}$ detectors will give $P_{N}$, the outcome of a NOON state projection measurement. Notice that state projection methods have been proposed before [3, 4, 5]. But those methods require $2 N$ photons for the generation of $N$-photon NOON state.

For a special case of $\mathrm{N}=4$, the actual implementation of the NOON state measurement is shown in Fig.2. The operators of the four detectors have the following form

$$
\left\{\begin{array}{l}
\hat{b}_{1}=\left(\hat{a}_{H}-\hat{a}_{V}\right) / 2+\hat{b}_{01} \\
\hat{b}_{2}=\left(\hat{a}_{H}+\hat{a}_{V}\right) / 2+\hat{b}_{02} \\
\hat{b}_{3}=\left(\hat{a}_{H}-i \hat{a}_{V}\right) / 2+\hat{b}_{03} \\
\hat{b}_{4}=\left(\hat{a}_{H}+i \hat{a}_{V}\right) / 2+\hat{b}_{04}
\end{array}\right.
$$

Here $\hat{b}_{0 n}(n=1-4)$ are some operators related to the vacuum modes $\hat{a}_{0 H, V}$ in the unused beam splitter input port and make no contribution to photon detection.

\section{ORTHOGONAL PROJECTION}

Let us now consider an input state of $|2,2\rangle$ for the NOON state projection measurement. This state can be produced in nondegenerate parametric down-conversion (NPDC). According to the previous discussion, we should have $P_{4}(|2,2\rangle)=0$ because $|2,2\rangle$ is orthogonal to the NOON state. In practice, however, we do not exactly have the state $|2,2\rangle$ in NPDC. 


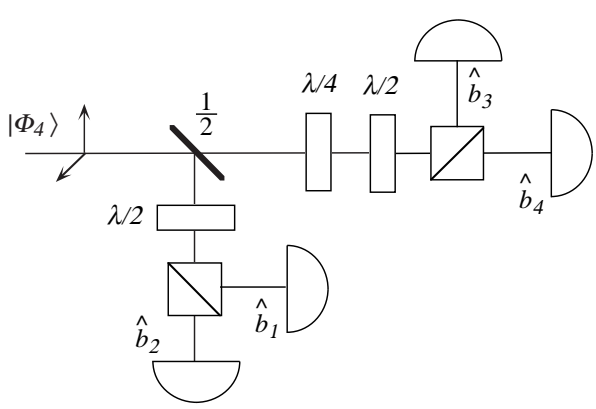

FIG. 2: NOON state projection measurement for $N=4 \cdot \lambda / 2$ is a half wave plate for 45 degree polarization rotation whereas $\lambda / 4$ is a quarter wave plate.

\section{A. Simple Pictures for Two Independent Pairs and Four Entangled Photons}

Usually in parametric down-conversion, a pair of photons is generated with extremely short correlation time $\left(T_{c} \sim 100 \mathrm{fs}\right)$ between the two photons but different pairs are produced completely in random as shown in Fig.3a. This case of well separated distinguishable two pairs of photons is called a $2 \times 2$ case. On the other hand, when ultra short pump pulses are applied to ensure the two pairs be produced within the short pump pulse duration, the four photons form an indistinguishable four-photon entangled state, as in Fig. 3 b. This case is called a $4 \times 1$ case. Sometimes, the paths between the two correlated photons (within one pair of photons) may not be balanced. Then all four photons are well separated and we call this situation a $1 \times 4$ case, as shown in Fig.3c.

(a)

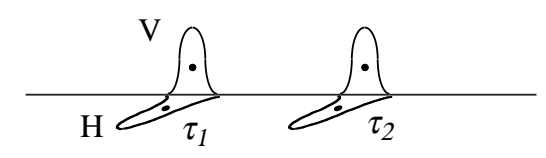

(b)

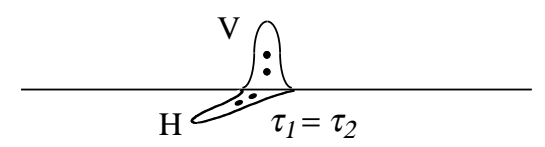

(c)

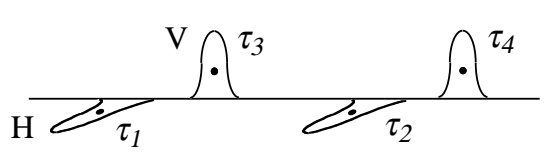

FIG. 3: Two pairs of photons from a Type-II parametric down-conversion: (a) two pairs are not generated in the same time and are distinguishable in time (the $2 \times 2$ case); (b) two pairs are indistinguishable (the $4 \times 1$ case); (c) all four photons are distinguishable (the $1 \times 4$ case).

Let's now label the times at which the two pairs are generated as $\tau_{1}, \tau_{2}$, respectively. For the case in Fig.3a
$(2 \times 2$ case $)$, we have $\left|\tau_{1}-\tau_{2}\right|>>T_{c}$ but for Fig. $3 \mathrm{~b}(4 \times 1$ case), $\left|\tau_{1}-\tau_{2}\right|<<T_{c}$. We can then write the quantum state of the two pairs as

$$
|\Phi\rangle=\left|\phi\left(\tau_{1}\right)\right\rangle \otimes\left|\phi\left(\tau_{2}\right)\right\rangle
$$

with

$$
|\phi\rangle=|H V\rangle
$$

Consider the detection scheme in Fig. 2 for $N=4$ case. The four-photon coincidence is proportional to the fourphoton correlation function:

$$
\begin{aligned}
& G^{(4)}\left(t_{1}, t_{2}, t_{3}, t_{4}\right) \\
& \quad=\| \hat{E}_{1}\left(t_{1}\right) \hat{E}_{2}\left(t_{2}\right) \hat{E}_{3}\left(t_{3}\right) \hat{E}_{4}\left(t_{4}\right)|\Phi\rangle \|^{2},
\end{aligned}
$$

where

$$
\left\{\begin{array}{l}
\hat{E}_{1}(t)=\left[\hat{E}_{H}(t)+\hat{E}_{V}(t)\right] / \sqrt{2}, \\
\hat{E}_{2}(t)=\left[\hat{E}_{H}(t)-\hat{E}_{V}(t)\right] / \sqrt{2}, \\
\hat{E}_{3}(t)=\left[\hat{E}_{H}(t)+i \hat{E}_{V}(t)\right] / \sqrt{2}, \\
\hat{E}_{4}(t)=\left[\hat{E}_{H}(t)-i \hat{E}_{V}(t)\right] / \sqrt{2},
\end{array}\right.
$$

where $\hat{E}_{H, V}(t)=\hat{a}_{H, V}(t)$ for the single mode treatment. When applying the above to the state in Eq.(13), we find six nonzero terms in Eq. (15), that is, when (i) $t_{1}=t_{2}=$ $\tau_{1}, t_{3}=t_{4}=\tau_{2}$ or $t_{1}=t_{2}=\tau_{2}, t_{3}=t_{4}=\tau_{1}$; (ii) $t_{1}=$ $t_{3}=\tau_{1}, t_{2}=t_{4}=\tau_{2}$ or $t_{1}=t_{3}=\tau_{2}, t_{2}=t_{4}=\tau_{1}$; (iii) $t_{1}=t_{4}=\tau_{1}, t_{2}=t_{3}=\tau_{2}$ or $t_{1}=t_{4}=\tau_{2}, t_{2}=t_{3}=\tau_{1}$. The case (i) can be calculated as

$$
\begin{aligned}
& \hat{E}_{1}\left(\tau_{1}\right) \hat{E}_{2}\left(\tau_{1}\right) \hat{E}_{3}\left(\tau_{2}\right) \hat{E}_{4}\left(\tau_{2}\right)|\Phi\rangle \\
& \quad=\hat{E}_{1}\left(\tau_{1}\right) \hat{E}_{2}\left(\tau_{1}\right)\left|\phi\left(\tau_{1}\right)\right\rangle \otimes \hat{E}_{3}\left(\tau_{2}\right) \hat{E}_{4}\left(\tau_{2}\right)\left|\phi\left(\tau_{2}\right)\right\rangle \\
& \quad=(1 / 2)^{2}(1-1)(i-i)|0\rangle=0 .
\end{aligned}
$$

It is the same for $\hat{E}_{1}\left(\tau_{2}\right) \hat{E}_{2}\left(\tau_{2}\right) \hat{E}_{3}\left(\tau_{1}\right) \hat{E}_{4}\left(\tau_{1}\right)|\Phi\rangle$. The zero result in this case stems from the two-photon Hong-OuMandel effect between $E_{1}$ and $E_{2}$ and between $E_{3}$ and $E_{4}$. Similarly, the case (ii) gives

$$
\begin{aligned}
& \hat{E}_{1}\left(\tau_{1}\right) \hat{E}_{2}\left(\tau_{2}\right) \hat{E}_{3}\left(\tau_{1}\right) \hat{E}_{4}\left(\tau_{2}\right)|\Phi\rangle \\
& \quad=\hat{E}_{1}\left(\tau_{2}\right) \hat{E}_{2}\left(\tau_{1}\right) \hat{E}_{3}\left(\tau_{2}\right) \hat{E}_{4}\left(\tau_{1}\right)|\Phi\rangle \\
& \quad=(1 / 2)^{2}(1+i)(1+i)|0\rangle
\end{aligned}
$$

and the case (iii) gives

$$
\begin{aligned}
& \hat{E}_{1}\left(\tau_{1}\right) \hat{E}_{2}\left(\tau_{2}\right) \hat{E}_{3}\left(\tau_{2}\right) \hat{E}_{4}\left(\tau_{1}\right)|\Phi\rangle \\
& \quad=\hat{E}_{1}\left(\tau_{2}\right) \hat{E}_{2}\left(\tau_{1}\right) \hat{E}_{3}\left(\tau_{1}\right) \hat{E}_{4}\left(\tau_{2}\right)|\Phi\rangle \\
& \quad=(1 / 2)^{2}(1-i)(1-i)|0\rangle
\end{aligned}
$$

When the two pairs are separated, i.e., $\left|\tau_{1}-\tau_{2}\right|>>T_{c}$, all six contributions are distinguishable $(2 \times 2$ case $)$ and we add their absolute values to give $G^{(4)}$ :

$$
G^{(4)}(2 \times 2)=2 \times 0+2\left|\frac{1+i}{2}\right|^{4}+2\left|\frac{1-i}{2}\right|^{4}=1 .
$$


On the other hand, when the two pairs overlap and become indistinguishable, i.e., $\left|\tau_{1}-\tau_{2}\right|<<T_{c}(4 \times 1$ case $)$, we add the six amplitudes:

$$
G^{(4)}(4 \times 1) \propto\left|2\left(\frac{1+i}{2}\right)^{2}+2\left(\frac{1-i}{2}\right)^{2}\right|^{2}=0 .
$$

The complete disappearance of $G^{(4)}(4 \times 1)$ is a result of orthogonality of $|2 H, 2 \mathrm{~V}\rangle$ with a NOON state. Thus the projection measurement gives a null result.

Notice that even when $\left|\tau_{1}-\tau_{2}\right|>>T_{c}$, there is still two-photon interference $(2 \times 2$ case $)$. So we expect that the value in Eq.(20) is smaller than the case when all four photons are well separated in time $(1 \times 4$ case $)$, as in Fig. 3c. In this case, the state of the four photons is

$$
\left|\Phi^{\prime}\right\rangle=\left|H\left(\tau_{1}\right) H\left(\tau_{2}\right) V\left(\tau_{3}\right) V\left(\tau_{4}\right)\right\rangle
$$

After expanding Eq. (15) by substituting Eq.(16), we find that only terms of the form $\hat{E}_{H} \hat{E}_{H} \hat{E}_{V} \hat{E}_{V} / 4$ or its permutations have nonzero values operating on $\left|\Phi^{\prime}\right\rangle$. There are 6 such terms. When each term operates on $\left|\Phi^{\prime}\right\rangle$, say, $\left.\hat{E}_{H}\left(t_{1}\right) \hat{E}_{H}\left(t_{2}\right) \hat{E}_{V}\left(t_{3}\right) \hat{E}_{V}\left(t_{4}\right)\left|H\left(\tau_{1}\right) H\left(\tau_{2}\right)\right| V\left(\tau_{3}\right) V\left(\tau_{4}\right)\right\rangle$, different permutations between $t_{1}=\tau_{1}, t_{2}=\tau_{2}$ or between $t_{3}=\tau_{3}, t_{4}=\tau_{4}$ give $2 \times 2=4$ contributions. Because of the distinguishability in time, all of them are incoherent to each other and they add together by their intensities (absolute value squares). So overall, we have $G^{(4)}(1 \times 4)=(1 / 4)^{2} \times 6 \times 4=3 / 2$.

As can be seen, $G^{(4)}(1 \times 4)>G^{(4)}(2 \times 2)>G^{(4)}(4 \times$ $1)$. This means that when we adjust the path difference between $\mathrm{H}$ and $\mathrm{V}, G^{(4)}$ will drop from $G^{(4)}(1 \times 4)$ to $G^{(4)}(2 \times 2)$ for the $2 \times 2$ case and to $G^{(4)}(4 \times 1)$ for the $4 \times 1$ case. This is somewhat similar to the Hong-OuMandel effect but for four photons, two from each side. Hence, the visibility of the generalized Hong-Ou-Mandel dip is

$$
\begin{aligned}
\mathcal{V}(2 \times 2) & =\left[G^{(4)}(1 \times 4)-G^{(4)}(2 \times 2)\right] / G^{(4)}(1 \times 4) \\
& =(3 / 2-1) /(3 / 2)=1 / 3
\end{aligned}
$$

for $2 \times 2$ case. For the $4 \times 1$ case, because $G^{(4)}(4 \times 1)=0$, we always have $\mathcal{V}(4 \times 1)=1$.

Although the above pictures is straightforward and easy to understand, it is not rigorous and it only applies to the two extreme cases. In the following, we will use a multi-mode theory of parametric down-conversion to accurately calculate the four-fold coincidence rate and confirm the results above.

\section{B. Multi-mode treatment}

The multi-mode theory for a type-II parametric downconversion gives the quantum state in the form of [22]

$$
|\Phi\rangle=|0\rangle+\eta\left|\Phi_{2}\right\rangle+\frac{1}{2} \eta^{2}\left|\Phi_{4}\right\rangle
$$

where

$$
\left|\Phi_{2}\right\rangle=\int d \omega_{1} d \omega_{2} \Phi\left(\omega_{1}, \omega_{2}\right) \hat{a}_{H}^{\dagger}\left(\omega_{1}\right) \hat{a}_{V}^{\dagger}\left(\omega_{2}\right)|0\rangle,
$$

and

$$
\begin{array}{r}
\left|\Phi_{4}\right\rangle=\int d \omega_{1} d \omega_{2} d \omega_{1}^{\prime} d \omega_{2}^{\prime} \Phi\left(\omega_{1}, \omega_{2}\right) \Phi\left(\omega_{1}^{\prime}, \omega_{2}^{\prime}\right) \\
\hat{a}_{H}^{\dagger}\left(\omega_{1}\right) \hat{a}_{V}^{\dagger}\left(\omega_{2}\right) \hat{a}_{H}^{\dagger}\left(\omega_{1}^{\prime}\right) \hat{a}_{V}^{\dagger}\left(\omega_{2}^{\prime}\right)|0\rangle .
\end{array}
$$

$\left|\Phi_{2}\right\rangle$ is a two-photon state and $\left|\Phi_{4}\right\rangle$ is a four-photon state.

Now let us calculate the four-photon correlation function $G^{(4)}$ in Eq. (15) with the state in Eq.(24). After expanding the product in Eq.(15) with Eq.(16), we find only the following combinations are non-zero:

$$
\begin{gathered}
\hat{E}_{1}\left(t_{1}\right) \hat{E}_{2}\left(t_{2}\right) \hat{E}_{3}\left(t_{3}\right) \hat{E}_{4}\left(t_{4}\right)|\Phi\rangle \\
=[(H H V V-V V H H)+ \\
+i(V H V H+H V H V)- \\
\quad-i(H V V H+V H H V)]|\Phi\rangle,
\end{gathered}
$$

where $H=\hat{E}_{H}, V=\hat{E}_{V}$ and we keep the time ordering. For a multi-mode state in Eq.(24), $\hat{E}_{H}, \hat{E}_{V}$ are expressed in multi-mode as

$$
\hat{E}_{H, V}(t)=\int d \omega \hat{a}_{H, V}(\omega) e^{-i \omega t},
$$

where $\hat{a}_{H, V}(\omega)$ is the annihilation operator satisfying the commutation relation:

$$
\left[\hat{a}^{\dagger}(\omega), \hat{a}\left(\omega^{\prime}\right)\right]=\delta\left(\omega-\omega^{\prime}\right)
$$

We can see that only the four-photon term in Eq. (24) will contribute to Eq.(27). The first term in Eq.(27) can be easily calculated as

$$
\begin{aligned}
& H H V V|\Phi\rangle \\
& \begin{array}{l}
=\eta^{2} \int d \omega_{1} d \omega_{2} d \omega_{1}^{\prime} d \omega_{2}^{\prime} \Phi\left(\omega_{1}, \omega_{2}\right) \Phi\left(\omega_{1}^{\prime}, \omega_{2}^{\prime}\right) \times \\
\quad \times\left(e^{-i \omega_{1} t_{1}-i \omega_{1}^{\prime} t_{2}}+e^{-i \omega_{1} t_{2}-i \omega_{1}^{\prime} t_{1}}\right) \times \\
\quad \times\left(e^{-i \omega_{2} t_{3}-i \omega_{2}^{\prime} t_{4}}+e^{-i \omega_{2} t_{4}-i \omega_{2}^{\prime} t_{3}}\right)|0\rangle \\
=2 \eta^{2}\left[g\left(t_{1}, t_{3}\right) g\left(t_{2}, t_{4}\right)+g\left(t_{1}, t_{4}\right) g\left(t_{2}, t_{3}\right)\right]|0\rangle,
\end{array}
\end{aligned}
$$

where

$$
g\left(t, t^{\prime}\right)=\int d \omega_{1} d \omega_{2} \Phi\left(\omega_{1}, \omega_{2}\right) e^{-i \omega_{1} t-i \omega_{2} t^{\prime}} .
$$

The rest of the terms in Eq.(27) have the following forms

$$
\begin{aligned}
& V V H H|\Phi\rangle \\
& \quad=2 \eta^{2}\left[g\left(t_{3}, t_{1}\right) g\left(t_{4}, t_{2}\right)+g\left(t_{3}, t_{2}\right) g\left(t_{4}, t_{1}\right)\right]|0\rangle
\end{aligned}
$$

$$
\begin{aligned}
& H V H V|\Phi\rangle \\
& \quad=2 \eta^{2}\left[g\left(t_{1}, t_{2}\right) g\left(t_{3}, t_{4}\right)+g\left(t_{1}, t_{4}\right) g\left(t_{3}, t_{2}\right)\right]|0\rangle,
\end{aligned}
$$


$V H V H|\Phi\rangle$
$\quad=2 \eta^{2}\left[g\left(t_{2}, t_{1}\right) g\left(t_{4}, t_{3}\right)+g\left(t_{2}, t_{3}\right) g\left(t_{4}, t_{1}\right)\right]|0\rangle$

$$
\begin{aligned}
& H V V H|\Phi\rangle \\
& \quad=2 \eta^{2}\left[g\left(t_{1}, t_{2}\right) g\left(t_{4}, t_{3}\right)+g\left(t_{1}, t_{3}\right) g\left(t_{4}, t_{2}\right)\right]|0\rangle,
\end{aligned}
$$

$$
\begin{aligned}
& V H H V|\Phi\rangle \\
& \quad=2 \eta^{2}\left[g\left(t_{2}, t_{1}\right) g\left(t_{3}, t_{4}\right)+g\left(t_{2}, t_{4}\right) g\left(t_{3}, t_{1}\right)\right]|0\rangle
\end{aligned}
$$

Next, let us assume the symmetry of $\Phi\left(\omega_{1}, \omega_{2}\right)=$ $\Phi\left(\omega_{2}, \omega_{1}\right)$ when the delay between $\mathrm{H}$ and $\mathrm{V}$ is zero. so that $g\left(t, t^{\prime}\right)=g\left(t^{\prime}, t\right)$. Then Eq. (27) becomes

$$
\begin{aligned}
& \hat{E}_{1}\left(t_{1}\right) \hat{E}_{2}\left(t_{2}\right) \hat{E}_{3}\left(t_{3}\right) \hat{E}_{4}\left(t_{4}\right)|\Phi\rangle \\
& \quad=4 \eta^{2}\left[g\left(t_{1}, t_{4}\right) g\left(t_{2}, t_{3}\right)-g\left(t_{1}, t_{3}\right) g\left(t_{2}, t_{4}\right)\right]|0\rangle,
\end{aligned}
$$

and Eq. 15ecomes

$$
\begin{aligned}
& G^{(4)}\left(t_{1}, t_{2}, t_{3}, t_{4}\right) \\
& \quad=16|\eta|^{4}\left|g\left(t_{1}, t_{4}\right) g\left(t_{2}, t_{3}\right)-g\left(t_{1}, t_{3}\right) g\left(t_{2}, t_{4}\right)\right|^{2} .
\end{aligned}
$$

The four-photon coincidence probability is proportional to an integral of $G^{(4)}$ with respect to all times:

$$
\begin{aligned}
P_{4}(0) & \propto \int_{-\infty}^{+\infty} d t_{1} d t_{2} d t_{3} d t_{4} G^{(4)}\left(t_{1}, t_{2}, t_{3}, t_{4}\right) \\
& =32|\eta|^{4}(\mathcal{A}-\mathcal{E}),
\end{aligned}
$$

where

$$
\begin{aligned}
& \mathcal{A}=\int_{-\infty}^{+\infty} d t_{1} d t_{2} d t_{3} d t_{4}\left|g\left(t_{1}, t_{4}\right) g\left(t_{2}, t_{3}\right)\right|^{2} \\
&= \int d \omega_{1} d \omega_{2} d \omega_{1}^{\prime} d \omega_{2}^{\prime}\left|\Phi\left(\omega_{1}, \omega_{2}\right) \Phi\left(\omega_{1}^{\prime}, \omega_{2}^{\prime}\right)\right|^{2} \\
& \mathcal{E}=\int d \omega_{1} d \omega_{2} d \omega_{1}^{\prime} d \omega_{2}^{\prime} \Phi\left(\omega_{1}, \omega_{2}\right) \times \\
& \quad \times \Phi\left(\omega_{1}^{\prime}, \omega_{2}^{\prime}\right) \Phi^{*}\left(\omega_{1}, \omega_{1}^{\prime}\right) \Phi^{*}\left(\omega_{2}, \omega_{2}^{\prime}\right) .
\end{aligned}
$$

When the delay between $\mathrm{H}$ and $\mathrm{V}$ is not zero, we may introduce a delay factor of $e^{i \omega_{2} \Delta T}$ for the $\mathrm{V}$-mode. Then $g\left(t, t^{\prime}\right)$ in Eq. 31] becomes $\bar{g}\left(t, t^{\prime}\right) \equiv g\left(t, t^{\prime}-\Delta T\right)$. Notice that now $\bar{g}\left(t, t^{\prime}\right) \neq \bar{g}\left(t^{\prime}, t\right)$ for nonzero $\Delta T$. Substituting $\bar{g}\left(t, t^{\prime}\right)$ into Eqs. 30 36) and carrying out the time integral in Eq. (39), we obtain after some lengthy calculation

$$
\begin{aligned}
& P_{4}(\Delta T) \propto 4|\eta|^{4}[ 12(\mathcal{A}+\mathcal{E})+4 \mathcal{E}^{(2)}(\Delta T)+ \\
&-8 \mathcal{E}_{1}^{(1)}(\Delta T)-8 \mathcal{E}_{2}^{(1)}(\Delta T)-8 \mathcal{E}_{3}^{(1)}(\Delta T) \\
&\left.+4 \mathcal{A}^{(2)}(\Delta T)-8 \mathcal{A}^{(1)}(\Delta T)\right]
\end{aligned}
$$

where

$$
\mathcal{A}^{(1)}(\tau)=A(\tau) A(0) \text { and } \mathcal{A}^{(2)}(\tau)=A^{2}(\tau),
$$

with

$$
A(\tau) \equiv \int d \omega_{1} d \omega_{2}\left|\Phi\left(\omega_{1}, \omega_{2}\right)\right|^{2} e^{i \omega_{2} \tau}
$$

and

$$
\begin{aligned}
& \mathcal{E}_{1}^{(1)}(\tau)=\int d \omega_{1} d \omega_{2} d \omega_{1}^{\prime} d \omega_{2}^{\prime} \Phi\left(\omega_{1}, \omega_{2}\right) \Phi\left(\omega_{1}^{\prime}, \omega_{2}^{\prime}\right) \times \\
& \times \Phi^{*}\left(\omega_{1}, \omega_{2}^{\prime}\right) \Phi^{*}\left(\omega_{1}^{\prime}, \omega_{1}\right) e^{i\left(\omega_{2}-\omega_{1}\right) \tau}, \\
& \mathcal{E}_{2}^{(1)}(\tau)=\int d \omega_{1} d \omega_{2} d \omega_{1}^{\prime} d \omega_{2}^{\prime} \Phi^{*}\left(\omega_{1}, \omega_{2}\right) \Phi^{*}\left(\omega_{1}^{\prime}, \omega_{2}^{\prime}\right) \times \\
& \times \Phi\left(\omega_{1}, \omega_{2}^{\prime}\right) \Phi\left(\omega_{1}^{\prime}, \omega_{2}\right) e^{i\left(\omega_{2}-\omega_{1}\right) \tau}, \\
& \mathcal{E}_{3}^{(1)}(\tau)=\int d \omega_{1} d \omega_{2} d \omega_{1}^{\prime} d \omega_{2}^{\prime} \Phi\left(\omega_{1}, \omega_{2}\right) \Phi\left(\omega_{1}^{\prime}, \omega_{2}^{\prime}\right) \times \\
& \times \Phi^{*}\left(\omega_{1}, \omega_{1}^{\prime}\right) \Phi^{*}\left(\omega_{2}, \omega_{2}^{\prime}\right) e^{i\left(\omega_{2}-\omega_{1}\right) \tau}, \\
& \mathcal{E}_{2}(\tau)=\int d \omega_{1} d \omega_{2} d \omega_{1}^{\prime} d \omega_{2}^{\prime} \Phi\left(\omega_{1}, \omega_{2}\right) \Phi\left(\omega_{1}^{\prime}, \omega_{2}^{\prime}\right) \times \\
& \times \Phi^{*}\left(\omega_{1}, \omega_{2}^{\prime}\right) \Phi\left(\omega_{1}^{\prime}, \omega_{2}\right) \times \\
& \times e^{i\left(\omega_{2}-\omega_{1}\right) \tau} e^{i\left(\omega_{2}^{\prime}-\omega_{1}^{\prime}\right) \tau} \text {. }
\end{aligned}
$$

Notice that $\mathcal{A}^{(1)}(0)=\mathcal{A}^{(2)}(0)=\mathcal{A}$ and $\mathcal{E}_{1}^{(1)}(0)=$ $\mathcal{E}_{2}^{(1)}(0)=\mathcal{E}_{3}^{(1)}(0)=\mathcal{E}^{(2)}(0)=\mathcal{E}$, and because of the symmetry $\Phi\left(\omega_{1}, \omega_{2}\right)=\Phi\left(\omega_{2}, \omega_{1}\right)$, we have $\mathcal{E}_{1}^{(1) *}(\tau)=\mathcal{E}_{2}^{(1)}(\tau)$ and $\mathcal{E}_{3}^{(1) *}(\tau)=\mathcal{E}_{3}^{(1)}(\tau)$.

When the delay $\Delta T$ is much larger than the coherence time, or the reciprocal of the bandwidth of $\Phi\left(\omega_{1}, \omega_{2}\right)$, there is no overlap among all four photons. This corresponds to the $1 \times 4$ case and all the $\Delta T$-dependent terms in Eq. (42) are zero. Hence we have $P_{4}$ at large delay as

$$
P_{4}(\infty) \propto 48|\eta|^{4}(\mathcal{A}+\mathcal{E}) .
$$

The visibility of the generalized Hong-Ou-Mandel dip is then

$$
\mathcal{V} \equiv \frac{P_{4}(\infty)-P_{4}(0)}{P_{4}(\infty)}=\frac{\mathcal{A}+5 \mathcal{E}}{3(\mathcal{A}+\mathcal{E})}
$$

Note that $\mathcal{E} \leq \mathcal{A}$ by Schwartz inequality. The equality stands if and only if $\Phi\left(\omega_{1}, \omega_{2}\right)$ is factorized as $\Phi\left(\omega_{1}, \omega_{2}\right)=$ $\phi\left(\omega_{1}\right) \phi\left(\omega_{2}\right)$. When $\mathcal{E}=0$, we have

$$
\mathcal{V}=1 / 3
$$

which is exactly same as Eq.(23) and corresponds to the situation when the two pairs of down-converted photons are well separated and independent of each other $(2 \times 2$ case). But when $\mathcal{E}=\mathcal{A}$, Eq.(50) becomes

$$
\mathcal{V}=1 \text {. }
$$

In this situation the two pairs of down-converted photons are overlapped to form an indistinguishable four-photon entangled state $(4 \times 1$ case $)$. 


\section{NOON STATE PROJECTION FOR DEMONSTRATION OF FOUR-PHOTON DE BROGLIE WAVELENGTH}

In this section, we will apply the NOON state projection measurement to two pairs of photons in EPR polarization entangled state of the form

$$
\left|\Phi^{+}\right\rangle=\frac{1}{\sqrt{2}}\left(|2 H\rangle+e^{2 i \varphi}|2 V\rangle\right) .
$$

When the pairs are overlapping and indistinguishable, it can be shown that the four-photon state has the form same as in Eq.(3) with the two modes denoting $\hat{a}_{H, V}$. If we make the NOON state projection measurement, only the NOON state part will contribute and the unwanted $|2,2\rangle$ state is projected out because it is orthogonal to the NOON state in Eq.(1).

Similar to the situation in Section III, we need to discuss the $2 \times 2$ case and the $4 \times 1$ case separately. We will start with the simple pictures that have been proven to be correct.

\section{A. Simple Pictures for Two Independent Pairs and Four Entangled Photons}

We again label the times at which the two pairs are generated as $\tau_{1}, \tau_{2}$, respectively. For the case in Fig.3a $(2 \times 2$ case $)$, we have $\left|\tau_{1}-\tau_{2}\right|>>T_{c}$ but for Fig. $3 \mathrm{~b}$, $\left|\tau_{1}-\tau_{2}\right|<<T_{c}(4 \times 1)$. We can then write the quantum state of the two pairs as

$$
|\Phi\rangle=\left|\Phi^{+}\left(\tau_{1}\right)\right\rangle \otimes\left|\Phi^{+}\left(\tau_{2}\right)\right\rangle,
$$

with $\left|\Phi^{+}\right\rangle$given in Eq. (53).

For the projection measurement in Fig.2 for $N=4$ case, the four-photon coincidence is proportional to the four-photon correlation function given in Eq. (15)

Similar to the case in previous section, when applying the above to the state in Eq. (54), we find six nonzero terms in Eq.(15), that is, when (i) $t_{1}=t_{2}=\tau_{1}, t_{3}=$ $t_{4}=\tau_{2}$ or $t_{1}=t_{2}=\tau_{2}, t_{3}=t_{4}=\tau_{1}$; (ii) $t_{1}=t_{3}=$ $\tau_{1}, t_{2}=t_{4}=\tau_{2}$ or $t_{1}=t_{3}=\tau_{2}, t_{2}=t_{4}=\tau_{1}$; (iii) $t_{1}=t_{4}=\tau_{1}, t_{2}=t_{3}=\tau_{2}$ or $t_{1}=t_{4}=\tau_{2}, t_{2}=t_{3}=\tau_{1}$. The case (i) can be calculated as

$$
\begin{aligned}
& \hat{E}_{1}\left(\tau_{1}\right) \hat{E}_{2}\left(\tau_{1}\right) \hat{E}_{3}\left(\tau_{2}\right) \hat{E}_{4}\left(\tau_{2}\right)|\Phi\rangle \\
& \quad=\hat{E}_{1}\left(\tau_{2}\right) \hat{E}_{2}\left(\tau_{2}\right) \hat{E}_{3}\left(\tau_{1}\right) \hat{E}_{4}\left(\tau_{1}\right)|\Phi\rangle \\
& \quad=\hat{E}_{1}\left(\tau_{1}\right) \hat{E}_{2}\left(\tau_{1}\right)\left|\Phi^{+}\left(\tau_{1}\right)\right\rangle \otimes \hat{E}_{3}\left(\tau_{2}\right) \hat{E}_{4}\left(\tau_{2}\right)\left|\Phi^{+}\left(\tau_{2}\right)\right\rangle \\
& \quad \propto\left(1-e^{i 2 \varphi}\right)\left(1+e^{i 2 \varphi}\right)|0\rangle
\end{aligned}
$$

Similarly, the case (ii) gives

$$
\begin{aligned}
& \hat{E}_{1}\left(\tau_{1}\right) \hat{E}_{2}\left(\tau_{2}\right) \hat{E}_{3}\left(\tau_{1}\right) \hat{E}_{4}\left(\tau_{2}\right)|\Phi\rangle \\
& \quad=\hat{E}_{1}\left(\tau_{2}\right) \hat{E}_{2}\left(\tau_{1}\right) \hat{E}_{3}\left(\tau_{2}\right) \hat{E}_{4}\left(\tau_{1}\right)|\Phi\rangle \\
& \quad \propto\left(1+i e^{i 2 \varphi}\right)\left(1+i e^{i 2 \varphi}\right)|0\rangle ;
\end{aligned}
$$

and the case (iii) gives

$$
\hat{E}_{1}\left(\tau_{1}\right) \hat{E}_{2}\left(\tau_{2}\right) \hat{E}_{3}\left(\tau_{2}\right) \hat{E}_{4}\left(\tau_{1}\right)|\Phi\rangle
$$

$$
\begin{aligned}
& =\hat{E}_{1}\left(\tau_{2}\right) \hat{E}_{2}\left(\tau_{1}\right) \hat{E}_{3}\left(\tau_{1}\right) \hat{E}_{4}\left(\tau_{2}\right)|\Phi\rangle \\
& \propto\left(1-i e^{i 2 \varphi}\right)\left(1-i e^{i 2 \varphi}\right)|0\rangle .
\end{aligned}
$$

When the two pairs are separated, i.e, $\left|\tau_{1}-\tau_{2}\right|>>T_{c}$, all three contributions are distinguishable and we add their absolute values to give $G^{(4)}$ :

$$
\begin{aligned}
G^{(4)} & \propto\left|\left(1-e^{i 4 \varphi}\right)\right|^{2}+\left|\left(1+i e^{i 2 \varphi}\right)\right|^{4}+\left|\left(1-i e^{i 2 \varphi}\right)\right|^{4} \\
& =14\left(1-\frac{3}{7} \cos 4 \varphi\right)
\end{aligned}
$$

On the other hand, when the two pairs overlap and become indistinguishable, i.e., $\left|\tau_{1}-\tau_{2}\right|<<T_{c}$, we add the three contributions in amplitudes:

$$
\begin{aligned}
G^{(4)} & \propto\left|\left(1-e^{i 4 \varphi}\right)+\left(1+i e^{i 2 \varphi}\right)^{2}+\left(1-i e^{i 2 \varphi}\right)^{2}\right|^{2} \\
& =18(1-\cos 4 \varphi)
\end{aligned}
$$

In both cases, the four-photon coincidence measurement has sinusoidal modulation with $4 \varphi$ - typical of 4 -photon de Broglie wave. But the first case only has $3 / 7=42 \%$ visibility but the second case produces $100 \%$ visibility, a result from a true NOON state projection.

We next consider more rigorously the multi-mode theory.

\section{B. Multi-mode treatment}

There are many ways to produce two-photon entangled state in Eq.(53). The straightforward way is to use two collinear degenerate type-I parametric down-conversion processes in series but with their orientations orthogonal to each other. One of the process gives the $|2 H\rangle$ state while the other produces $|2 V\rangle$. If the two processes are pumped from a common source, the final state will be in the form of Eq. (53). In the multi-mode theory, the quantum state for system up to the four-photon has the form of

$$
\begin{aligned}
|\Phi\rangle=|0\rangle & +\eta_{1}\left|\Phi_{H 2}\right\rangle+\eta_{2}\left|\Phi_{V 2}\right\rangle+\frac{1}{2}\left(\eta_{1}^{2}\left|\Phi_{H 4}\right\rangle+\right. \\
& \left.+\eta_{2}^{2}\left|\Phi_{V 4}\right\rangle+2 \eta_{1} \eta_{2}\left|\Phi_{H 2}\right\rangle\left|\Phi_{V 2}\right\rangle\right)
\end{aligned}
$$

where

$$
\left|\Phi_{M 2}\right\rangle=\int d \omega_{1} d \omega_{2} \Phi\left(\omega_{1}, \omega_{2}\right) \hat{a}_{M}^{\dagger}\left(\omega_{1}\right) \hat{a}_{M}^{\dagger}\left(\omega_{2}\right)|0\rangle,
$$

and

$$
\begin{array}{r}
\left|\Phi_{M 4}\right\rangle=\int d \omega_{1} d \omega_{2} d \omega_{1}^{\prime} d \omega_{2}^{\prime} \Phi\left(\omega_{1}, \omega_{2}\right) \Phi\left(\omega_{1}^{\prime}, \omega_{2}^{\prime}\right) \\
\hat{a}_{M}^{\dagger}\left(\omega_{1}\right) \hat{a}_{M}^{\dagger}\left(\omega_{2}\right) \hat{a}_{M}^{\dagger}\left(\omega_{1}^{\prime}\right) \hat{a}_{M}^{\dagger}\left(\omega_{2}^{\prime}\right)|0\rangle
\end{array}
$$

where $M=H, V .\left|\Phi_{M 2}\right\rangle$ is a two-photon state and $\left|\Phi_{M 4}\right\rangle$ is a four-photon state.

Now let us calculate the four-photon correlation function $G^{(4)}$ in Eq. (15) with the state in Eq. (60). After 
expanding the product in Eq.(15) with Eq.(16), we find only the following combinations are non-zero:

$$
\begin{aligned}
& \hat{E}_{1}\left(t_{1}\right) \hat{E}_{2}\left(t_{2}\right) \hat{E}_{3}\left(t_{3}\right) \hat{E}_{4}\left(t_{4}\right)|\Phi\rangle \\
& =(H H-V V)(H H+V V)|\Phi\rangle+ \\
& \quad+i(V H-H V)(V H-H V)|\Phi\rangle
\end{aligned}
$$

where $H=\hat{E}_{H}, V=\hat{E}_{V}$ and we keep the time ordering. The first term in Eq. 63] can be easily calculated as

$$
\begin{aligned}
& (H H-V V)(H H+V V)|\Phi\rangle= \\
& =\left(\eta_{1}^{2} H H H H\left|\Phi_{H 4}\right\rangle-\eta_{2}^{2} V V V V\left|\Phi_{V 4}\right\rangle\right) / 2+ \\
& \quad+\eta_{1} \eta_{2}(H H V V-V V H H)\left|\Phi_{H 2}\right\rangle\left|\Phi_{V 2}\right\rangle \\
& =\left\{\left(\eta_{1}^{2}-\eta_{2}^{2}\right) F\left(t_{1}, t_{2}, t_{3}, t_{4}\right) / 2+\right. \\
& \left.\quad+2 \eta_{1} \eta_{2}\left[g\left(t_{1}, t_{2}\right) g\left(t_{3}, t_{4}\right)-g\left(t_{3}, t_{4}\right) g\left(t_{1}, t_{2}\right)\right]\right\}|0\rangle \\
& =\left[\left(\eta_{1}^{2}-\eta_{2}^{2}\right) F\left(t_{1}, t_{2}, t_{3}, t_{4}\right) / 2\right]|0\rangle,
\end{aligned}
$$

where $g\left(t, t^{\prime}\right)$ is given in Eq. (31) and

$$
\begin{gathered}
F\left(t_{1}, t_{2}, t_{3}, t_{4}\right) \\
\equiv\left\langle 0\left|\hat{E}_{H}\left(t_{1}\right) \hat{E}_{H}\left(t_{2}\right) \hat{E}_{H}\left(t_{3}\right) \hat{E}_{H}\left(t_{4}\right)\right| \Phi_{H 4}\right\rangle \\
=8\left[g\left(t_{1}, t_{2}\right) g\left(t_{3}, t_{4}\right)+g\left(t_{1}, t_{3}\right) g\left(t_{2}, t_{4}\right)+\right. \\
\left.+g\left(t_{1}, t_{4}\right) g\left(t_{3}, t_{2}\right)\right] .
\end{gathered}
$$

Here we assumed the symmetry $\Phi\left(\omega_{1}, \omega_{2}\right)=\Phi\left(\omega_{2}, \omega_{1}\right)$ for type-I PDC so that $g\left(t, t^{\prime}\right)=g\left(t^{\prime}, t\right)$.

For the second term in Eq. [63), there are four contributions after expanding the product and each can be calculated as in Eq.(64). We then have

$$
\begin{aligned}
& (V H-H V)(V H-H V)|\Phi\rangle \\
& \quad=\eta_{1} \eta_{2}(V H-H V)(V H-H V)\left|\Phi_{H 2}\right\rangle\left|\Phi_{V 2}\right\rangle \\
& \quad=8 \eta_{1} \eta_{2}\left[g\left(t_{1}, t_{3}\right) g\left(t_{2}, t_{4}\right)-g\left(t_{1}, t_{4}\right) g\left(t_{2}, t_{3}\right)\right]|0\rangle
\end{aligned}
$$

where we used $g\left(t, t^{\prime}\right)=g\left(t^{\prime}, t\right)$. Combining Eqs. 6466), we have

$$
\begin{aligned}
& \hat{E}_{1}\left(t_{1}\right) \hat{E}_{2}\left(t_{2}\right) \hat{E}_{3}\left(t_{3}\right) \hat{E}_{4}\left(t_{4}\right)|\Phi\rangle \\
& \quad=4\left[b_{1}\left(\eta_{1}^{2}-\eta_{2}^{2}\right)+2 i b_{2} \eta_{1} \eta_{2}\right]|0\rangle,
\end{aligned}
$$

with

$$
\begin{aligned}
b_{1} \equiv g\left(t_{1}, t_{2}\right) g\left(t_{1}, t_{2}\right)+ & g\left(t_{1}, t_{3}\right) g\left(t_{2}, t_{4}\right)+ \\
& +g\left(t_{1}, t_{4}\right) g\left(t_{3}, t_{2}\right)
\end{aligned}
$$

and

$$
b_{2} \equiv g\left(t_{1}, t_{3}\right) g\left(t_{2}, t_{4}\right)-g\left(t_{1}, t_{4}\right) g\left(t_{2}, t_{3}\right) .
$$

Next we assume $\eta_{1}=\eta$ and $\eta_{2}=\eta e^{2 i \varphi}$, where $\varphi$ is the phase difference between the vertical and horizontal down-converted photons. Then Eq. (15) becomes

$$
\begin{aligned}
& G^{(4)}\left(t_{1}, t_{2}, t_{3}, t_{4}\right) \\
& \quad=16|\eta|^{2}\left|b_{1}\left(1-e^{4 i \varphi}\right)+2 i b_{2} e^{2 i \varphi}\right|^{2} \\
& \quad=64|\eta|^{2}\left|b_{2}-b_{1} \sin 2 \varphi\right|^{2}
\end{aligned}
$$

After an integration of $G^{(4)}$ with respect to all time, we obtain the four-photon coincidence probability as

$$
P_{4} \propto \int_{-\infty}^{+\infty} d t_{1} d t_{2} d t_{3} d t_{4} G^{(4)}\left(t_{1}, t_{2}, t_{3}, t_{4}\right)
$$

$$
=64|\eta|^{2}\left(B_{2}+B_{1} \sin ^{2} 2 \varphi-2 B_{12} \sin 2 \varphi\right),
$$

with

$$
\begin{aligned}
& B_{1}=\int d t_{1} d t_{2} d t_{3} d t_{4}\left(\left|b_{1}\right|^{2}\right)=3(\mathcal{A}+2 \mathcal{E}) \\
& B_{2}=\int d t_{1} d t_{2} d t_{3} d t_{4}\left(\left|b_{2}\right|^{2}\right)=2(\mathcal{A}-\mathcal{E})
\end{aligned}
$$

and

$$
B_{12}=\int d t_{1} d t_{2} d t_{3} d t_{4}\left(b_{1} b_{2}\right)=0
$$

where $\mathcal{A}, \mathcal{E}$ are given in Eqs.40 41), respectively. Finally, we have

$$
P_{4} \propto 64|\eta|^{2}(\mathcal{E}+7 \mathcal{A} / 2)(1-\mathcal{V} \cos 4 \varphi),
$$

with

$$
\mathcal{V} \equiv \frac{3(\mathcal{A}+2 \mathcal{E})}{7 \mathcal{A}+2 \mathcal{E}}
$$

When $\mathcal{E}=\mathcal{A}$, we have

$$
P_{4} \propto 18(1-\cos 4 \varphi)
$$

which is exactly same as Eq.(59) and corresponds to the situation when the two pairs overlap to form an indistinguishable four-photon entangled state. However, when $\mathcal{E}=0$, Eq.(75) becomes

$$
P_{4} \propto 14\left(1-\frac{3}{7} \cos 4 \varphi\right)
$$

which is same as Eq.(58) and in this situation the two pairs of down-converted photons are well separated and independent of each other.

\section{CONCLUSION AND DISCUSSION}

From the above calculation, we find that the projection measurement scheme that we designed in Section II has no contribution from the non-NOON states due to orthogonal projection. Thus it extracts out the contribution only from the NOON state. The essence of the orthogonal projection is a multi-photon interference where amplitudes instead of intensities are added. The outcome of the measurement is highly dependent on the multi-photon entanglement in the quantum state. From the visibility, we can then quantitatively define the degree of multi-photon entanglement. This is some issue we will discuss elsewhere.

Although the scheme discussed in Section IV is somewhat similar to the scheme by Walther et al [7], there are some fundamental differences in the two schemes. First, the starting quantum states are different: we use two 
collinear type-I parametric down-conversion processes to produce a state of the form in Eq.(3) whereas the scheme by Walther et al starts with two pairs of photons in EPR state. Secondly, the measurement schemes are different: ours is a NOON state projection measurement but the measurement by Walther et al is a special arrangement for the cancellation of the unwanted terms. Of course, the end result is the same: only the NOON state contributes to the measurement. Thirdly, the phase variations in the scheme of Walther et al are locally separated whereas in our scheme, the phases are all together giving a true $4 \varphi$ dependence.

From the construction process for the NOON state projection measurement, we find that the method can be generalized to an arbitrary projection measurement of N-photon superposition states in Eq. (8) since it relies on the factorization or finding the roots of a polynomial equation:

$$
\sum_{n=0}^{N} c_{n} x^{N-n} y^{n}=0,
$$

which always has solution.

In the discussion throughout the paper, we assumed that the spatial modes of the two polarizations are perfectly matched. In a real experiment, however, misalignment will result in imperfect spatial mode match and lead to a reduced visibility in any interference. The effect of misalignment can be incorporated in our formulism by introducing a spatial factor of $e^{i k_{H} x}$ or $e^{i k_{V} x}$ in Eq. (28) 22]. For simplicity, we only consider a misalignment in propagation direction for the spatial mismatch. More complicated cases of spatial mode match will have similar result. Thus we can use one dimensional model where $x$ is the coordinate of the detector along the direction of $\mathbf{k}_{H}-\mathbf{k}_{V}$. Four detectors will have four different coordinates. The final result will be an integral over each detector's size of $\Delta x$ (we assume all four detectors have the same size) and this will lead to a non-unit visibility of $v=\operatorname{sinc}(\pi \Delta x / L)$ for single photon interference at each detector with $L \equiv \lambda / \Delta \theta$ as the single photon interference fringe spacing ( $\Delta \theta$ is the angle between $\mathbf{k}_{H}$ and $\mathbf{k}_{V}$ ). Two-photon interference visibility will be $v_{2}=v^{2}$. With the spatial dependent factors inserted in Eq.(28), we may go through similar calculation on the temporal integrals and carry out the spatial integral. It is straightforward to show that the four-photon interference visibility in Eq.(501) is changed to

$$
\mathcal{V}=\frac{2 v_{2}(\mathcal{A}+3 \mathcal{E})-v_{2}^{2}(\mathcal{A}+\mathcal{E})}{3(\mathcal{A}+\mathcal{E})}
$$

and Eq.(76) to

$$
\mathcal{V}=\frac{3(\mathcal{A}+2 \mathcal{E}) v_{2}^{2}}{\left(6+v_{2}^{2}\right) \mathcal{A}+2 \mathcal{E}\left(3-2 v_{2}\right)}
$$

where $v_{2}=v^{2}=\operatorname{sinc}^{2}(\pi \Delta x / L)$ is the two-photon interference visibility.

\section{Acknowledgments}

This work was funded by National Fundamental Research Program (2001CB309300), the Innovation funds from Chinese Academy of Sciences, and National Natural Science Foundation of China (Grant No. 60121503). ZYO is also supported by the US National Science Foundation under Grant No. 0245421.
[1] J. J. Bollinger, W. M. Itano, D. J. Wineland, and D. J. Heinzen, Phys. Rev. A 54, R4649 (1996).

[2] Z. Y. Ou, Phys. Rev. A 55, 2598 (1997).

[3] Hwang Lee, Pieter Kok, Nicolas J. Cerf, and Jonathan P. Dowling, Phys. Rev. A 65, 030101(R) (2002).

[4] Pieter Kok, Hwang Lee, and Jonathan P. Dowling, Phys. Rev. A 65, 052104 (2002).

[5] Jaromir Fiurasek, Phys. Rev. A 65, 053818 (2002).

[6] H. F. Hofmann, Phys. Rev. A 70, 023812 (2004).

[7] P. Walther, J.-W. Pan, M. Aspelmeyer, R. Ursin, S. Gasparoni, and A. Zeilinger, Nature (London) 429, 158 (2004).

[8] M. W. Mitchell, J. S. Lundeen, and A. M. Steinberg, Nature (London) 429, 161 (2004).

[9] F. Shafiei, P. Srinivasan, and Z. Y. Ou, Phys. Rev. A 70, 043803 (2004).

[10] Haibo Wang and Takayoshi Kobayashi, Phys. Rev. A 71, 021802(R) (2005).

[11] W. Heisenberg, Z. Phys. 43, 172 (1927).

[12] Z. Y. Ou, X. Y. Zou, L. J. Wang, and L. Mandel, Phys. Rev. A 42, 2957 (1990).

[13] J. G. Rarity, P. R. Tapster, E. Jakeman, T. Larchuk, R.
A. Campos, M. C. Teich, and B. E. A. Saleh, Phys. Rev. Lett. 65, 1348 (1990).

[14] E. J. S. Fonseca, C. H. Monken, and S. Padua, Phys. Rev. Lett. 82, 2868 (1999).

[15] Keiichi Edamatsu, Ryosuke Shimizu, and Tadashi Itoh, Phys. Rev. Lett. 89, 213601 (2002).

[16] Agedi N. Boto, Pieter Kok, Daniel S. Abrams, Samuel L. Braunstein, Colin P. Williams, and Jonathan P. Dowling, Phys. Rev. Lett. 85, 2733 (2000).

[17] Milena DAngelo, Maria V. Chekhova, and Yanhua Shih, Phys. Rev. Lett. 87, 013602 (2001).

[18] C. K. Hong, Z. Y. Ou, and L. Mandel, Phys. Rev. Lett. 59, 2044 (1987).

[19] Z. Y. Ou, J.-K. Rhee, and L. J. Wang, Phys. Rev. Lett. 83, 959 (1999).

[20] K. Tsujino, H. F. Hofmann, S. Takeuchi, and K. Sasaki, Phys. Rev. Lett. 92, 153602 (2004).

[21] Z. Y. Ou, Phys. Rev. A 72053814 (2005).

[22] Z. Y. Ou, J.-K. Rhee, and L. J. Wang, Phys. Rev. A 60, 593 (1999). 